\title{
Relationships of Alpha-SMA-Positive Fibroblasts and SDF-1-Positive Tumor Cells with Neoangiogenesis in Nasopharyngeal Carcinoma
}

\author{
Shumin Wang, ${ }^{1,2}$ Ning Ma, ${ }^{3}$ Shosuke Kawanishi, ${ }^{4}$ Yusuke Hiraku, ${ }^{1}$ Shinji Oikawa, \\ Ying Xie, ${ }^{2}$ Zhe Zhang, ${ }^{2}$ Guangwu Huang, ${ }^{2}$ and Mariko Murata ${ }^{1}$ \\ ${ }^{1}$ Department of Environmental and Molecular Medicine, Mie University Graduate School of Medicine, 2-174 Edobashi, \\ Tsu, Mie 514-8507, Japan \\ ${ }^{2}$ Department of Otolaryngology Head and Neck Surgery, First Affiliated Hospital of Guangxi Medical University, \\ No. 22 Shuangyong Road, Nanning 530027, China \\ ${ }^{3}$ Faculty of Nursing Science, Suzuka University of Medical Science, 3500-3 Minamitamagaki-cho, Suzuka, Mie 513-8670, Japan \\ ${ }^{4}$ Faculty of Pharmaceutical Sciences, Suzuka University of Medical Science, 3500-3 Minamitamagaki-cho, Suzuka, Mie 513-8670, Japan
}

Correspondence should be addressed to Mariko Murata; mmurata@doc.medic.mie-u.ac.jp

Received 13 December 2013; Revised 28 February 2014; Accepted 8 April 2014; Published 27 April 2014

Academic Editor: Jeroen Rouwkema

Copyright (C) 2014 Shumin Wang et al. This is an open access article distributed under the Creative Commons Attribution License, which permits unrestricted use, distribution, and reproduction in any medium, provided the original work is properly cited.

Nasopharyngeal carcinoma (NPC) is one of the most prevalent malignant tumors with poor prognosis in Southern China and Southeast Asia. Angiogenesis-related molecules can be promising therapeutic targets in NPC. To investigate the relationships of cancer-associated fibroblasts (CAFs) and chemokine-related molecules with neoangiogenesis, we compared immunohistochemical analyses of alpha-smooth-muscle actin ( $\alpha$-SMA), stroma-derived factor-1 (SDF-1), and its receptor CXCR4 in primary NPC specimens and chronic nasopharyngitis tissues. In addition, we examined the expression of vascular endothelial growth factor (VEGF-A), and CD133- and VEGF- receptor-2 (VEGFR-2) double positive cells, as endothelial progenitor cells (EPCs). We also assessed CD34-positive microvessels. Significantly higher expression of $\alpha$-SMA was observed in fibroblasts in NPC stroma. The immunoreactive intensities of SDF-1 and CXCR4 were significantly higher in NPC cells. CXCR4-positive cells and CD133/VEGFR2- double positive cells were observed in the stroma surrounding cancer nests, and VEGF was detected in both cancer and stromal cells. Microvessel density was significantly higher in the stroma of NPC tissues compared to chronic nasopharyngitis tissues. Our data suggest that CAFs and NPC tumor cells may enhance neoangiogenesis in a VEGF- and SDF-1-dependent manner by recruiting EPCs from the bone marrow into tumor stroma.

\section{Introduction}

Genetic and cell biology studies indicate that tumor growth is determined not only by malignant cancer cells but also by the tumor stroma [1]. Fibroblasts in the tumor stroma acquire a perpetually activated phenotype and become a subpopulation that can be identified by the expression of $\alpha$ smooth-muscle actin ( $\alpha$-SMA) [2]. $\alpha$-SMA-positive fibroblasts are called cancer-associated fibroblasts (CAFs) [2]. Many reports have indicated that CAFs are important promoters of tumor growth and progression, as their production of growth factors and chemokines and extracellular matrix facilitate the angiogenic recruitment of endothelial cells and pericytes [24].

Stroma-derived factor-1 (SDF-1) is an $\alpha$-chemokine that binds only to the receptor CXCR4. Recent studies have suggested that the SDF-1/CXCR4 axis plays an important and unique role in the egress of hematopoietic stem/ progenitor cells from bone marrow [5, 6]. Hematopoietic stem/progenitor cells are recruited into the cancer stroma, where they become endothelial progenitor cells (EPCs). CD133 is a hematopoietic stem/progenitor cell marker [7] 
and unexpressed on mature differentiated endothelial cells [8]. Vascular endothelial growth factor receptor-2 (VEGFR2), also known as Flk1, is an early endothelial marker [9]. CD34 also includes endothelial antigens [10]. The double positive cells of CD133 with VEGFR-2 or CD34 are verified as EPCs [8], which may contribute to angiogenesis and tumor development [11].

Microvessel density measurement is a quantitative method of assessing angiogenesis [12]. CD34 can be used as an indicator of microvessel density [10]. Tumor microvessel density is correlated with the concentration and expression of proangiogenic growth factors such as vascular endothelial growth factor (VEGF) [13]. VEGF-A, a 34- to 46-kDa glycoprotein, is a potent stimulator of endothelial cell growth, and it can stimulate both physiological and pathological angiogeneses [14].

Nasopharyngeal carcinoma (NPC) is one of the most prevalent malignant tumors in Southern China and Southeast Asia, and its prognosis has been poor for decades [15]. Therefore, a better understanding of its pathogenesis is needed. Tumor progression clearly depends on angiogenesis [16], and, thus, targeting tumor angiogenesis is a promising strategy for NPC and many solid tumors [17]. To investigate the relationships of cancer-associated fibroblasts (CAFs) and related molecules to neoangiogenesis, we performed immunohistochemical analyses of alpha-smooth-muscle actin ( $\alpha$-SMA), stroma-derived factor-1 (SDF-1), and its cognate receptor CXCR4 in primary NPC lesions. We also examined EPCs by double fluorescent staining of CD133 with VEGFR-2 and CD34. In addition, to evaluate neoangiogenesis, we observed microvessels by CD34 staining and their growth factor VEGF-A.

\section{Material and Methods}

2.1. Patients. Formalin-fixed and paraffin-embedded biopsies were obtained from 57 patients $(45.2 \pm 10.7$ years old, 38 males and 19 females) with NPC, and chronic nasopharyngitis tissues were obtained via tonsillectomy from 31 patients $(40.1 \pm 11.9$ years old, 20 males and 11 females) with chronic nasopharyngitis, who functioned as normal controls. All subjects were patients at the Department of Otolaryngology-Head and Neck Surgery, First Affiliated Hospital of Guangxi Medical University, Nanning, China, and provided informed consent prior to participation. Diagnoses were made by experienced pathologists according to the World Health Organization (WHO) classification. The pathological diagnosis of all NPC samples was nonkeratinizing carcinoma. This study was performed in accordance with ethical review committee approval notice (2009-07-07) of the First Affiliated Hospital of Guangxi Medical University, China, and ethical approval (number 1116) by Mie University, Japan. We removed identifying information from all samples before analysis.

2.2. Immunoperoxidase Study of $\alpha$-SMA, CXCR4, VEGF-A, and $C D 34$. Standard immunoperoxidase methods were used to examine the distribution of $\alpha$-SMA, CXCR4, VEGF$\mathrm{A}$, and CD34 in NPC tissues and normal controls. After deparaffinization and rehydration, antigen was retrieved in $5 \%$ urea buffer by microwave heating for $5 \mathrm{~min}$ and then incubated in $1 \% \mathrm{H}_{2} \mathrm{O}_{2}$ for $30 \mathrm{~min}$ to block endogenous peroxidase activity. Sections of $3 \mu \mathrm{m}$ thickness were incubated overnight at room temperature with the following antibodies: rabbit polyclonal anti- $\alpha$-SMA (1:200, Abcam, Cambridge, MA), rabbit monoclonal anti-CXCR4 (1:100, Abcam), rabbit polyclonal anti-VEGF (human VEGF-A165, 1:200, Abcam), and mouse monoclonal anti-CD34 (1:200, Monosan, Uden, Netherlands). For the rabbit antibodies ( $\alpha$-SMA, CXCR 4 , and VEGF-A), the sections were incubated with goat anti-rabbit IgG for $3 \mathrm{~h}$, then incubated with peroxidase antiperoxidase complex for $2 \mathrm{~h}$. For mouse antibody (CD34), the sections were incubated with biotinylated anti-mouse IgG for $3 \mathrm{~h}$ then incubated with avidin-biotin complex (Vectastain ABC kit, Vector Laboratories, Burlingame, CA) for $2 \mathrm{~h}$. Sections were then incubated with 3,3'-diaminobenzidine (DAB substrate kit; Vector Laboratories). Nuclei were counterstained by hematoxylin.

\subsection{Immunofluorescence Study of SDF-1, CD133, VEGFR-2,} and CD34. Immunoreactivities of SDF-1 in nasopharyngeal tissues were assessed by single immunofluorescence labeling study. Double positive cells of CD133 with VEGFR-2 or CD34 were detected by double immunofluorescence labeling studies. Briefly, deparaffinized and rehydrated sections $(3-\mu \mathrm{m}$ thickness) were incubated with 5\% skim milk and were then incubated with mouse monoclonal anti-SDF-1 (1:100, Santa Cruz Biotechnology, CA), mouse monoclonal anti-CD133 (1:100, Abgent Inc., San Diego, CA), rabbit monoclonal anti-CD133 (1:100, Abcam), rabbit polyclonal anti-VEGFR2 (1:50, Abcam), or mouse monoclonal anti-CD34 (1:200, Monosan) as the primary antibody overnight at room temperature. The sections were then incubated for $3 \mathrm{~h}$ with Alexa 594-labeled goat antibody against rabbit IgG or Alexa 488labeled goat antibody against mouse IgG (1:400) (Molecular Probes, Eugene, OR). Stained sections were examined using fluorescence microscopy (BX53, Olympus, Tokyo, Japan).

2.4. Immunohistochemical Grading. Immunohistochemical (IHC) grading based on intensity and frequency of staining results was performed by two independent investigators without knowledge of the patients' clinicopathological features. The staining intensity was scored as negative $(0)$, weak $(+1)$, moderate $(+2)$, or strong $(+3)$. Frequency of positive cells in specific areas was scored as negative (0), less than $25 \%$ $(+1), 25-50 \%(+2), 51-75 \%(+3)$, or more than $75 \%(+4)$. IHC grades were assigned by multiplying the intensity score by the frequency score as follows: -, absent expression (0); +, weak expression (1); ++, moderate expression (2); +++, high expression (3); or ++++ , very high expression (4).

2.5. Microvessel Evaluation. Microvessel density (MVD) was estimated using a light microscope. We checked MDV by two methods; one was counting number of microvessels and the other was digital microscopy assessment. Prior to 

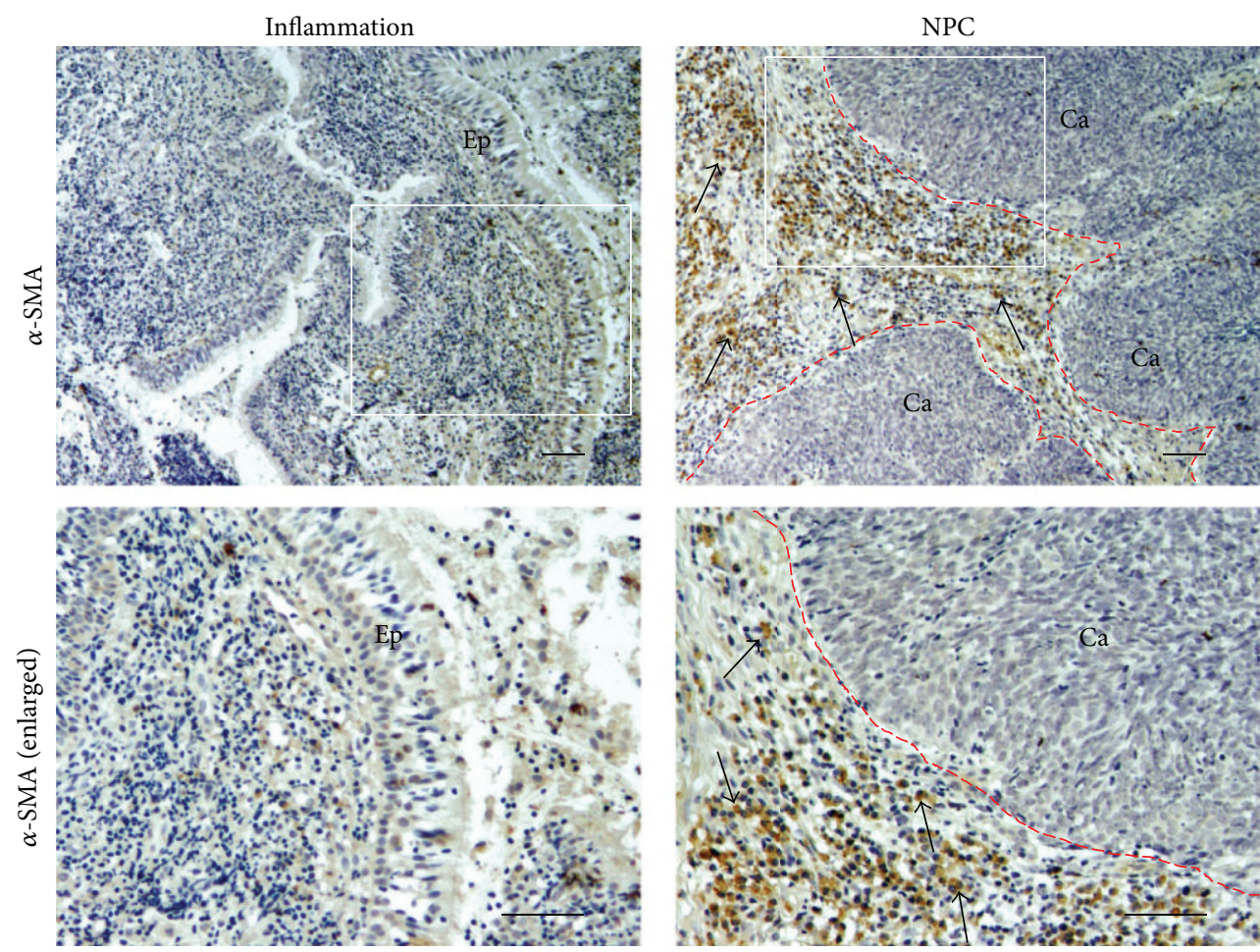

FIGURE 1: The expression of $\alpha$-SMA in nasopharyngeal tissues. Formalin-fixed and paraffin-embedded biopsies of nasopharyngeal tissues were obtained from chronic nasopharyngitis (inflammation) and NPC tissues. The expression of $\alpha$-SMA was assessed by immunoperoxidase staining (brown). Arrows indicate $\alpha$-SMA-positive cells. The red dotted line is border between cancer nest and stromal area. Original magnification is 100x (upper) and the insets are enlarged in the lower panels (200x). Scale bar represents $50 \mu \mathrm{m}$. Ep: epithelium. Ca: cancer cells.

counting the microvessels, slides were examined at low power magnification $(\times 40)$ to identify the area with the highest density of the microvessels. The five most vascular fields within the section were selected, and MVD measurement was performed at high power magnification $(\times 200)$. For counting microvessels, areas staining for CD34, whether single endothelial cells or clusters of endothelial cells, regardless of the absence/presence of a lumen were counted as individual microvessels. The microvessel numbers of the five most vascular areas were averaged to give an estimate of tissue microvessel density for each patient. For digital microscopy assessment of MVD, the intensity and area of endothelial staining were quantitatively measured using the cellSens Standard Ver1.4 Imaging Software (Olympus, Tokyo, Japan). The immunohistochemical stain was then selected using the "color selection" function and the "area/density (intensity) measurement" functions were used to calculate the respective values.

2.6. Statistical Analysis. Statistical differences were determined by the chi-square test and Mann-Whitney $U$ test. $P<$ 0.05 was considered to be statistically significant. Correlation between factors was assessed with the Spearman correlation test. Statistical analysis was performed using SPSS19 for Windows.

\section{Results}

3.1. Expression of $\alpha-S M A$ in the Stroma of NPC Tissues. Figure 1 shows the localization of $\alpha$-SMA in nasopharyngeal tissues from patients with NPC and chronic nasopharyngitis (inflammation). In the chronic nasopharyngitis samples, $\alpha$ SMA expression was scarce and was detected only in vascular pericytes and vascular smooth muscle (data not shown). No immunoreactivity was observed in normal nasopharyngeal epithelium or stromal fibroblasts. In contrast, tumor cells showed negative immunoreactivity for $\alpha$-SMA, but $\alpha$-SMApositive stromal cells (arrows) were observed surrounding cancer nests. $\alpha$-SMA was expressed in the cytoplasm of fibroblasts in primary NPC tissues. In most of the NPC cases, a large proportion of fibroblasts were $\alpha$-SMA positive.

\subsection{Expression of SDF-1 and Its Receptor CXCR4 in Nasopha-} ryngeal Tissues. Figure 2 shows the expression patterns of cancer-specific cytokine SDF-1 and its receptor CXCR4 in primary NPC and chronic nasopharyngitis tissues. In chronic nasopharyngitis tissues (inflammation), epithelial cells showed weak SDF-1 and CXCR4 immunoreactivity (Figures 2(a) and 2(b), left). In primary NPC tissues, SDF-1 was intensively expressed in the cytoplasm and membrane of NPC tumor cells (Figure 2(a), right) and mucosal epithelial 

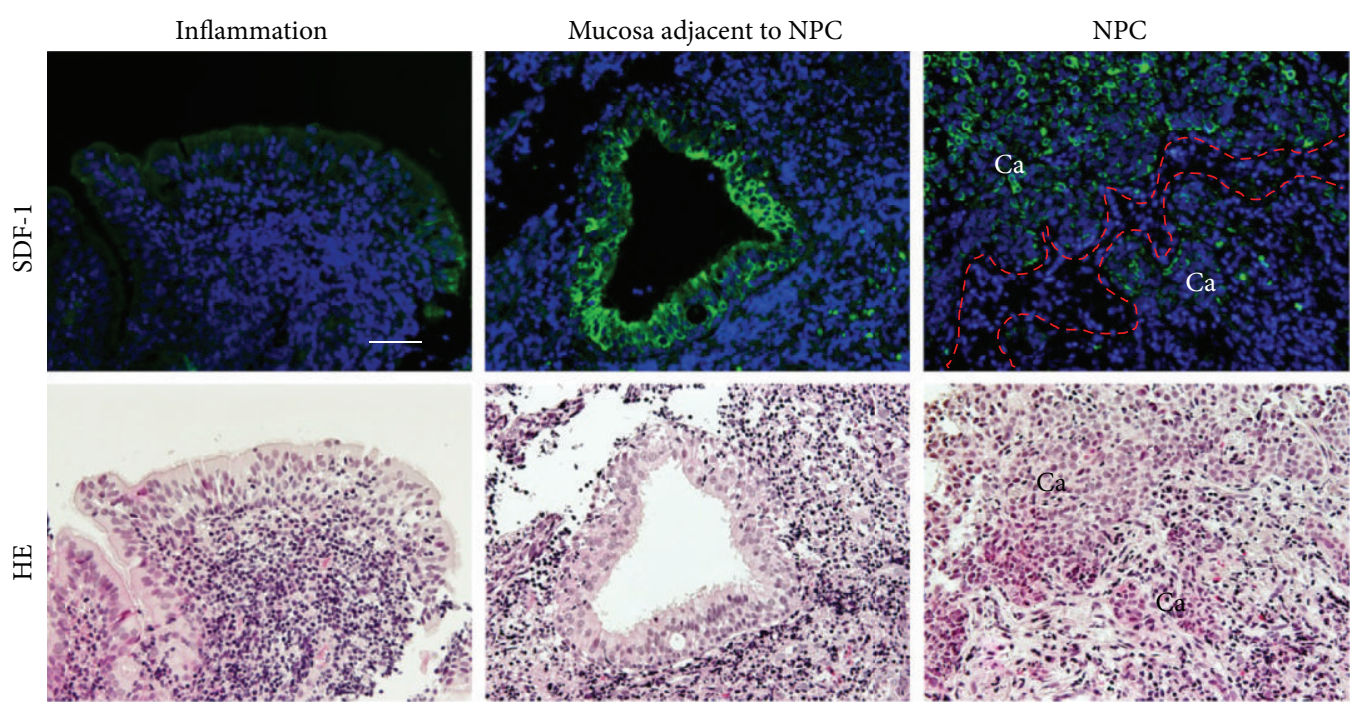

(a)
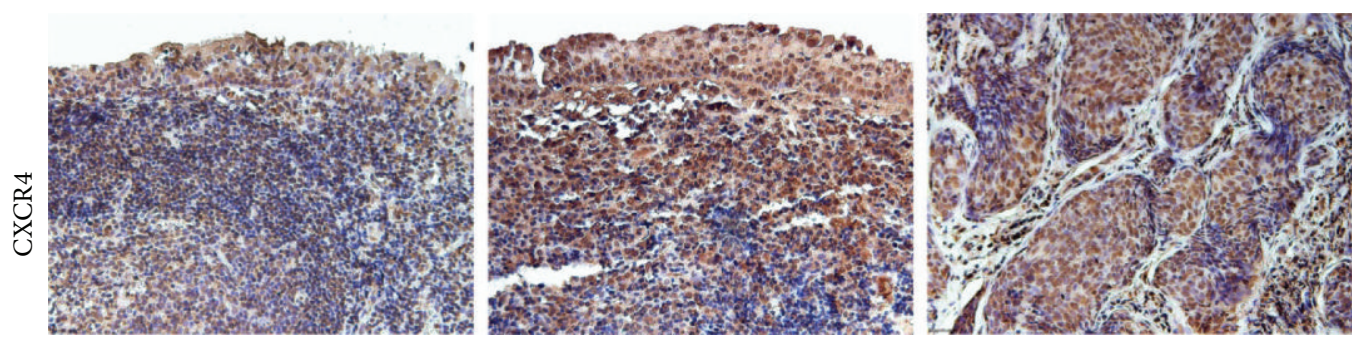

(b)

FIGURE 2: The expression of SDF-1 and CXCR4 in chronic nasopharyngitis (inflammation) and NPC tissues. (a) The expression of SDF-1 was assessed by immunofluorescence staining (green). Nuclei were counterstained by DAPI (blue). The red dotted line is border between cancer nest and stromal area. HE staining of parallel sections. (b) Immunoperoxidase staining of CXCR4 protein (brown). Original magnification is 200x. Scale bar represents $50 \mu \mathrm{m}$. Ca: cancer cells.

cells adjacent to NPC nest (Figure 2(a), middle) but not in the stromal cells (Figure 2(a), right). CXCR4 was strongly expressed in the nucleus, cytoplasm, and cellular membrane of NPC cells and mucosa adjacent to NPC nest (Figure 2(b), middle and right).

3.3. Expression of CD133 and VEGF-A in NPC Tissues. Figure 3 shows the expression patterns of angiogenesisrelated molecules in primary NPC and inflammatory tissues. Abundant CD133-positive cells were observed in the stroma of NPC (Figure 3(a), right) but not in nasopharyngitis tissues (Figure 3(a), left). Weak VEGF-A immunoreactivity was observed in the epithelial cells and stromal cells of inflammatory tissues (Figure 3(b), left). On the other hand, a predominantly membrane/cytoplasmic distribution was seen in both stromal cells and NPC cells (Figure 3(b), middle and right).

3.4. CD34-Positive Microvessels and Evaluation of MVD in NPC Tissues. Figure 3(c) shows the membrane and cytoplasm of vascular endothelial cells were stained with CD34 in the stroma of nasopharyngeal tissues. The microvessels in section labeled by anti-CD34 presented single endothelial cell or small clusters of the cells, with or without an irregular lumen (Figure 3(c), arrows). CD34-positive vessels were more abundant in the stromal tissue of NPC samples (Figure 3(c), middle and right) than in inflammatory tissues (Figure 3(c), left), indicating higher formation of microvessels in NPC. Distribution of the microvessels within the tumor was uneven and heterogeneous. The area of highest vascularization was usually in the stroma between the NPC nests. CD34-positive cell clusters were counted as microvessel density at high power magnification $(\times 200)$. Number of microvessels/field was significantly higher in NPC stroma $(78.04 \pm 21.7)$ than inflammatory tissues $(42.9 \pm 17.6, P<$ 0.05). CD34-positive area fraction (\%) was significantly higher in NPC (6.45 \pm 4.68$)$ than inflammation $(0.3 \pm 0.36$, $P<0.01)$.

3.5. Expression of CD133 with VEGFR-2 or CD34 in Stromal Cells. Figure 4 shows stromal cells with CD133, VEGFR2 and CD34 immunoreactivities in NPC tissues. Double immunofluorescent staining of the stem/progenitor cell marker and endothelial marker showed a small number of CD133 and VEGFR-2 double positive cells in the stroma 

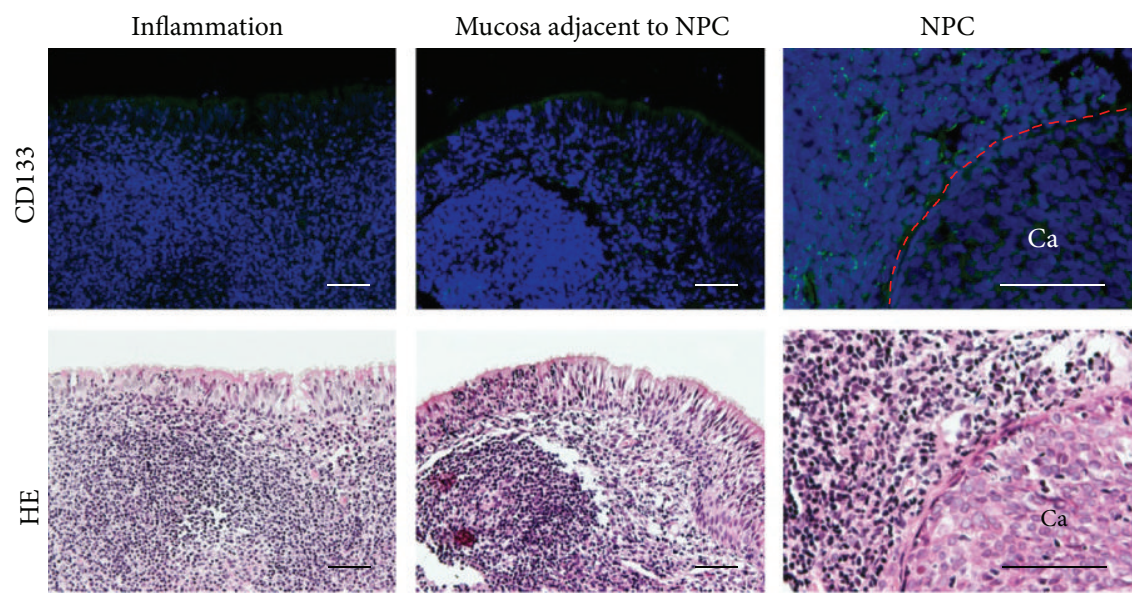

(a)
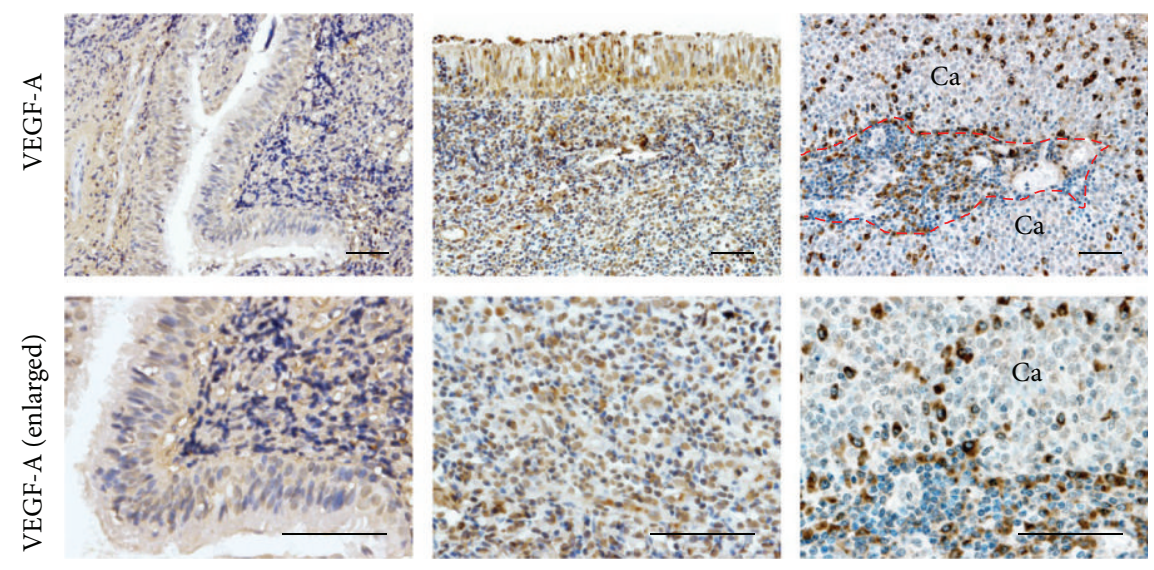

(b)
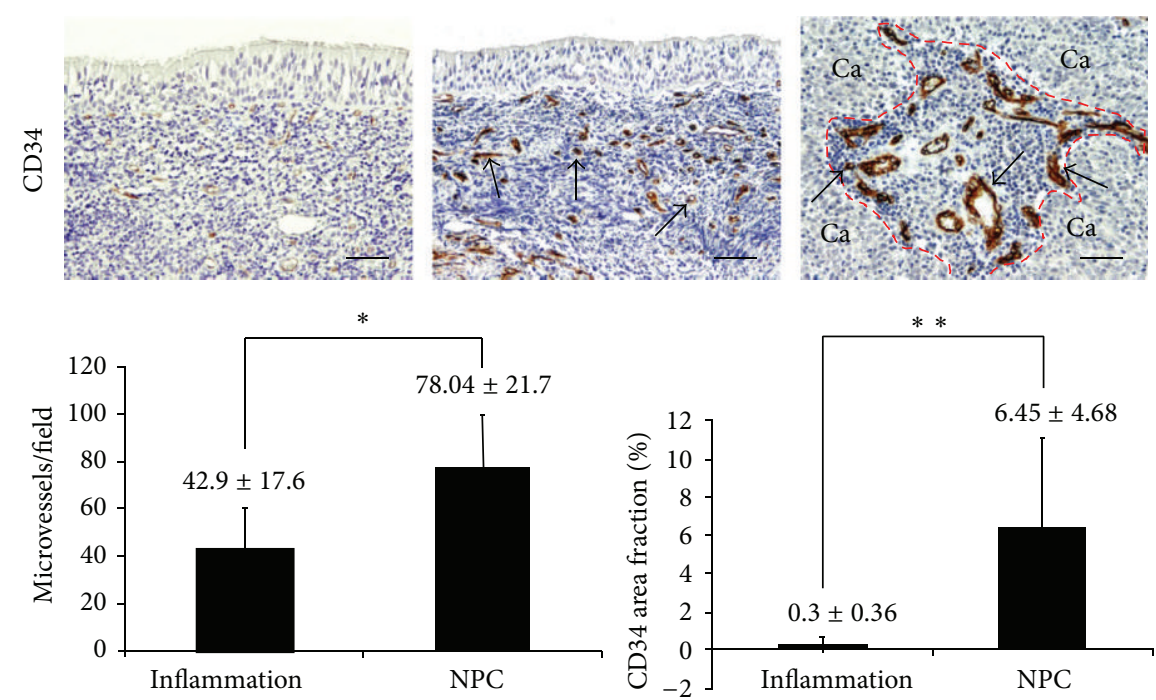

(c)

FIGURE 3: Staining patterns of CD133, VEGF-A, CD34, and microvessel density in chronic nasopharyngitis (inflammation) and NPC tissues. (a) The expression of CD133 by immunofluorescence staining (green). Nuclei were counterstained by DAPI (blue). HE staining of parallel sections. Immunoperoxidase staining of (b) VEGF-A (brown) and (c) CD34 (brown). Arrows show CD34-positive vessels. The red dotted line is border between cancer nest and stromal area. Original magnification is 200x (VEGF-A in upper panels, CD34) and VEGF-A enlarged in the lower panels $(400 \mathrm{x})$. Scale bar represents $50 \mu \mathrm{m}$. Ca: cancer cells. Graphs represent average and SD of microvessels/field and CD34 area fraction (\%) for inflammation $(n=6)$ and NPC tissues $(n=10) . P$ values were calculated by the Mann-Whitney $U$ test in comparison to chronic nasopharyngitis tissues. ${ }^{*} P<0.05 ;{ }^{* *} P<0.01$. 
TABLE 1: Expression of $\alpha$-SMA and related molecules in nasopharyngeal tissues.

\begin{tabular}{|c|c|c|c|c|c|c|c|c|c|}
\hline & \multicolumn{2}{|c|}{ Location } & \multirow{2}{*}{ Group } & \multicolumn{5}{|c|}{ 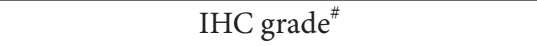 } & \multirow{2}{*}{$P$ value ${ }^{*}$} \\
\hline & Stroma & Cancer nest & & - & + & ++ & +++ & ++++ & \\
\hline \multirow{2}{*}{$\alpha$-SMA } & \multirow{2}{*}{ Positive $^{\$}$} & \multirow{2}{*}{ ND } & Chronic nasopharyngitis (21) & 11 & 7 & 2 & 1 & 0 & \multirow{2}{*}{0.000} \\
\hline & & & NPC (18) & 0 & 4 & 0 & 7 & 7 & \\
\hline \multirow{2}{*}{ CD133 } & \multirow{2}{*}{ Positive } & \multirow{2}{*}{ ND } & Chronic nasopharyngitis (10) & 9 & 1 & 0 & 0 & 0 & \multirow{2}{*}{0.006} \\
\hline & & & NPC $(30)$ & 8 & 9 & 9 & 4 & 0 & \\
\hline \multirow{2}{*}{ VEGFR-2 } & \multirow{2}{*}{ Positive } & \multirow{2}{*}{ ND } & Chronic nasopharyngitis (8) & 7 & 1 & 0 & 0 & 0 & \multirow{2}{*}{0.04} \\
\hline & & & NPC $(10)$ & 2 & 5 & 1 & 2 & 0 & \\
\hline \multirow{2}{*}{$\mathrm{CD} 34$} & \multirow{2}{*}{ Positive } & \multirow{2}{*}{ ND } & Chronic nasopharyngitis (14) & 0 & 8 & 6 & 0 & 0 & \multirow{2}{*}{0.000} \\
\hline & & & NPC $(24)$ & 0 & 0 & 3 & 7 & 14 & \\
\hline \multirow{2}{*}{ CXCR4 } & \multirow{2}{*}{ Positive } & \multirow{2}{*}{ Positive } & Chronic nasopharyngitis (6) & 0 & 3 & 1 & 2 & 0 & \multirow{2}{*}{0.041} \\
\hline & & & NPC (11) & 0 & 0 & 2 & 4 & 5 & \\
\hline \multirow{2}{*}{ VEGF-A } & \multirow{2}{*}{ Positive } & \multirow{2}{*}{ Positive } & Chronic nasopharyngitis (10) & 0 & 1 & 5 & 4 & 0 & \multirow{2}{*}{0.015} \\
\hline & & & NPC (19) & 0 & 0 & 2 & 9 & 8 & \\
\hline \multirow{2}{*}{ SDF-1 } & \multirow{2}{*}{ ND } & \multirow{2}{*}{ Positive } & Chronic nasopharyngitis (22) & 5 & 9 & 5 & 2 & 1 & \multirow{2}{*}{0.008} \\
\hline & & & NPC (20) & 3 & 0 & 7 & 5 & 5 & \\
\hline
\end{tabular}

\# IHC grades were assigned to each specimen according to the grade of staining intensity as described in Section 2 .

${ }^{*} P$ values were calculated by the chi-square test in comparison to chronic nasopharyngitis tissues.

${ }^{\$}$ IHC reactivity: positive; ND: not detected.
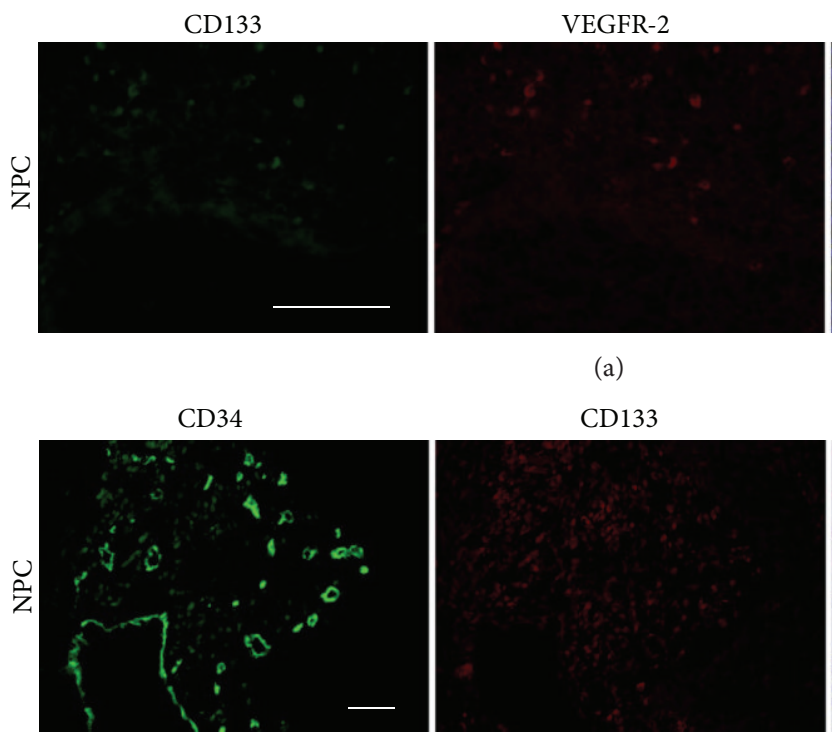

(b)

(a)

CD133
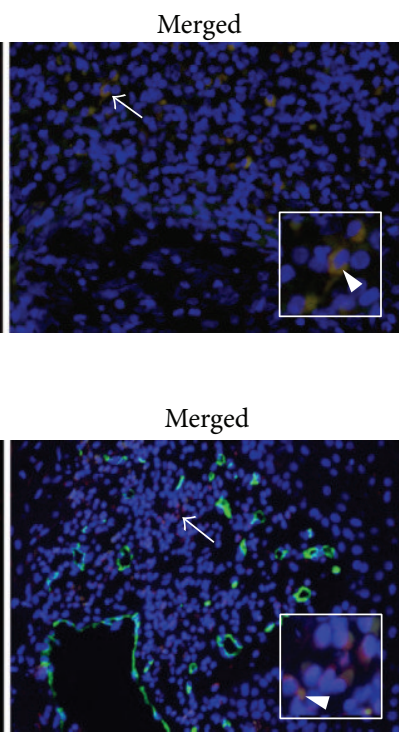

Figure 4: Double immunofluorescent staining of CD133 with VEGFR-2 and CD34 in NPC tissues. (a) The immunofluorescence expression of CD133 (green) and VEGFR-2 (red). (b) The immunofluorescence expression of CD34 (green) and CD133 (red). Nuclei were counterstained by DAPI (blue) in the merged pictures. Arrows and arrowheads indicate the double positive cells and the enlarged (insets). Original magnification is $400 \mathrm{x}$ (a) and 200x (b). Scale bar represents $50 \mu \mathrm{m}$.

surrounding NPC nests (Figure 4(a), merged, arrow, enlarged picture in inset, and arrowhead). We also observed double immunostaining of CD133 and CD34 in membrane and cytoplasm of some cells in the stroma (Figure 4(b), merged, arrow, enlarged picture in inset, and arrowhead).

3.6. Relationship of $\alpha$-SMA and Related Molecules in Nasopharyngeal Samples. Table 1 shows statistical analyses of the expression levels of $\alpha$-SMA and angiogenesis-related molecules with comparisons between NPC and chronic nasopharyngitis patients. The immunoreactivity of $\alpha$-SMA was significantly increased in NPC tissues relative to inflammatory tissues $(P<0.001)$. CD133, VEGFR-2, and CD34 also showed significantly higher expression in the stroma of primary NPC tissues compared with inflammatory tissues (CD133, $P=0.006$; VEGFR-2, $P=0.04$; CD34, $P<0.001$ ). 
CXCR4 and VEGF-A were observed in both stromal and cancer cells, and the IHC grades were significantly higher in NPC than chronic nasopharyngeal tissues (CXCR4, $P=0.041$; VEGF-A, $P=0.015)$. SDF-1 was highly expressed in cancer cells of NPC tissues than inflamed tissues (SDF-1, $P=0.008$ ). We found a significant correlation between $\alpha$-SMA and CD34 (Spearman rank correlation coefficient; $r=0.721, P<0.001)$ in nasopharyngeal tissues. SDF1 expression was also positively correlated with CD34 expression ( $r=0.701, P=0.002)$.

\section{Discussion}

In this study, we firstly found $\alpha$-SMA-positive fibroblasts in the stroma of NPC tissues. CAFs, activated fibroblasts that often express $\alpha$-SMA within desmoplastic lesions, are associated with cancer progression [2]. Their involvement has been reported in tumorigenesis and metastasis in several types of cancers $[3,18-21] . \alpha$-SMA was shown to be useful for predicting patient prognosis of pancreatic ductal adenocarcinoma [18]. In our study, we demonstrated that a large proportion of stromal cells surrounding cancer nests were $\alpha$-SMA positive in most of the NPC samples but not in the chronic nasopharyngitis samples, suggesting the involvement of CAFs in NPC progression.

Several studies showed high expression of SDF-1/CXCR4 in many cancers $[4,22-24]$, including NPC $[25,26]$. In this study, SDF-1 and its receptor CXCR4 were intensively expressed in primary NPC samples. SDF-1 was strongly expressed in NPC tumor cells and mucosal epithelial cells adjacent to NPC nest, but it was not expressed in stromal cells, including CAFs. Although SDF-1 is known to be released by CAFs in several cancers $[3,27,28]$, SDF-1 could be produced by cancer cells as a paracrine factor [24]. High expression of SDF-1 of tumor cells forms a local gradient of the chemokine in the tumor region, recruiting CXCR4-expressing bone marrow-derived progenitor cells to the tumor [11]. Our results suggested that NPC tumor cells secrete SDF-1 into the stroma and cancer cells themselves in paracrine and autocrine loops, and this chemokine may contribute to the process of vasculogenesis by recruiting CXCR4-expressing cells, such as inflammatory cells and endothelial progenitor cells, to the NPC mass.

We observed strong expression of VEGF-A in both stromal cells and tumor cells of NPC tissues, indicating that stromal cells and NPC cells can secrete significant amounts of VEGF. VEGF is believed to play an important role in angiogenesis. It is responsible for initiating capillary growth by attracting and stimulating endothelial cells in the reactive stroma [29]. Fibroblasts and inflammatory cells are the principal source of host-derived VEGF [30]. Cancer cells themselves can also release VEGF [31-33]. Critical steps in tumor angiogenesis include the recruitment of circulating endothelial progenitor cells [34] and vascular endothelial cell migration [35]. CAFs enhance tumor angiogenesis by inducing mobilization and recruitment of circulating endothelial progenitor cells $[2,3]$. Such endothelial progenitor cells likely enter the tumor stroma from the bone marrow via the peripheral circulation [36], and the recruitment is mediated in part by SDF-1 and VEGF [36]. Kryczek et al. suggested that tumor-derived SDF-1 and VEGF could synergize to stimulate vascular endothelial cell proliferation and protect against cell apoptosis [4]. Therefore, both SDF-1 and VEGF could contribute to angiogenesis in NPC.

Peichev et al. [8] found that incubation of $\mathrm{CD} 33^{+} \mathrm{VEGEF}^{+}{ }^{+}$cells, putative EPCs, with VEGF and other factors resulted in differentiation into mature $\mathrm{CD} 33^{-}{ }^{-}$EEGEF-2 ${ }^{+}$cells. Hwang et al. [37] characterized EPCs and endothelial cells by RT-PCR, and EPCs were positive for cell markers CD133 and CD34, and after differentiation into endothelial cells, they highly expressed CD34 but not CD133. Interestingly, in our study, double positive cells of CD133 with VEGFR-2 or CD34 were observed surrounding NPC nests, which may be consistent with the hypothesis [38] regarding circulating endothelial progenitor cells' mobilization and migration. Tumor angiogenesis is indispensable to tumor growth, invasion, and metastasis, and the current gold standard to characterize tumor angiogenesis is histological MVD technique [39]. Microvessel density is an outcome indicator in several malignancies [10, 40-42], including NPC [43]. In the present study, high microvessel density was obtained in NPC stroma and putative EPCs were observed near microvessels, suggesting the tumorassociated neoangiogenesis in NPC. Since concomitant chemoradiotherapy (i.e., cisplatin and 5-fluorouracil followed by radiation) is currently recommended for invasive NPC [44], antiangiogenic therapy in combination with cytotoxic therapies [45] would help to improve general treatment approaches for NPC. Our present study provides the possibility of targeting angiogenesis-related molecules and CAFs for treating NPC. Further studies are required for elucidating the mechanism of facilitating angiogenesis in nasopharyngeal carcinoma.

\section{Conclusions}

Our observations suggested that $\alpha$-SMA-positive fibroblasts (CAFs) and NPC tumor cells enhanced neoangiogenesis in a VEGF- and SDF-1-dependent manner.

\section{Abbreviations \\ NPC: Nasopharyngeal carcinoma \\ CAF: Cancer-associated fibroblast \\ $\alpha$-SMA: Alpha-smooth-muscle actin \\ SDF-1: Stroma-derived factor-1 \\ VEGF: Vascular endothelial growth factor \\ EPC: Endothelial progenitor cell \\ MVD: Microvessel density \\ IHC: Immunohistochemistry.}

\section{Conflict of Interests}

The authors declare that there is no conflict of interests regarding the publication of this paper. 


\section{Acknowledgments}

This work was partly supported by Grants-in-Aid for Scientific Research from the Ministry of Education, Culture, Sports, Science and Technology of Japan (Grant nos. 19406021, 22406016, and 25305020), Japan-China Research Cooperative Program (2010-2011), the Natural Science Foundation of China (81060167), and Program for New Century Excellent Talents in University (NCET-12-0654).

\section{References}

[1] R. Kalluri, "Basement membranes: structure, assembly and role in tumour angiogenesis," Nature Reviews Cancer, vol. 3, no. 6, pp. 422-433, 2003.

[2] R. Kalluri and M. Zeisberg, "Fibroblasts in cancer," Nature Reviews Cancer, vol. 6, no. 5, pp. 392-401, 2006.

[3] A. Orimo, P. B. Gupta, D. C. Sgroi et al., "Stromal fibroblasts present in invasive human breast carcinomas promote tumor growth and angiogenesis through elevated SDF-1/CXCL12 secretion," Cell, vol. 121, no. 3, pp. 335-348, 2005.

[4] I. Kryczek, A. Lange, P. Mottram et al., "CXCL12 and vascular endothelial growth factor synergistically induce neonaniogenisis in human ovarian cancers," Cancer Research, vol. 65, no. 2, pp. 465-472, 2005.

[5] Y. Gazitt and C. Akay, "Mobilization of myeloma cells involves SDF-1/CXCR4 signaling and downregulation of VLA-4," Stem Cells, vol. 22, no. 1, pp. 65-73, 2004.

[6] M. B. Dwinell, H. Ogawa, K. E. Barrett, and M. F. Kagnoff, "SDF-1/CXCL12 regulates cAMP production and ion transport in intestinal epithelial cells via CXCR4," American Journal of Physiology: Gastrointestinal and Liver Physiology, vol. 286, no. 5, pp. G844-G850, 2004.

[7] A. H. Yin, S. Miraglia, E. D. Zanjani et al., "AC133, a novel marker for human hematopoietic stem and progenitor cells," Blood, vol. 90, no. 12, pp. 5002-5012, 1997.

[8] M. Peichev, A. J. Naiyer, D. Pereira et al., "Expression of VEGFR2 and AC133 by circulating human CD34+ cells identifies a population of functional endothelial precursors," Blood, vol. 95, no. 3, pp. 952-958, 2000.

[9] I. Flamme, G. Breier, and W. Risau, "Vascular endothelial growth factor (VEGF) and VEGF receptor 2 (flk-1) are expressed during vasculogenesis and vascular differentiation in the quail embryo," Developmental Biology, vol. 169, no. 2, pp. 699712, 1995.

[10] F. Ajili, M. Kacem, H. Tounsi et al., "Prognostic impact of angiogenesis in nonmuscle invasive bladder cancer as defined by microvessel density after immunohistochemical staining for CD34," Ultrastructural Pathology, vol. 36, no. 5, pp. 336-342, 2012.

[11] U. M. Domanska, R. C. Kruizinga, W. B. Nagengast et al., "A review on CXCR4/CXCL12 axis in oncology: no place to hide," European Journal of Cancer, vol. 49, no. 1, pp. 219-230, 2013.

[12] I. Chebib, M. T. Shabani-Rad, M. S. Chow, J. Zhang, and Z. H. Gao, "Microvessel density and clinicopathologic characteristics in hepatocellular carcinoma with and without cirrhosis," Biomarker Insights, vol. 2, pp. 59-68, 2007.

[13] M. Toi, K. Inada, H. Suzuki, and T. Tominaga, “Tumor angiogenesis in breast cancer: its importance as a prognostic indicator and the association with vascular endothelial growth factor expression," Breast Cancer Research and Treatment, vol. 36, no. 2, pp. 193-204, 1995.
[14] R. Roskoski Jr., "Vascular endothelial growth factor (VEGF) signaling in tumor progression," Critical Reviews in Oncology/Hematology, vol. 62, no. 3, pp. 179-213, 2007.

[15] Q. Tao and A. T. C. Chan, "Nasopharyngeal carcinoma: molecular pathogenesis and therapeutic developments," Expert Reviews in Molecular Medicine, vol. 9, no. 12, pp. 1-24, 2007.

[16] J. Folkman, "Tumor angiogenesis: therapeutic implications," New England Journal of Medicine, vol. 285, no. 21, pp. 1182-1186, 1971.

[17] Y. Cao, Z. L. Zhang, M. Zhou et al., "Pericyte coverage of differentiated vessels inside tumor vasculature is an independent unfavorable prognostic factor for patients with clear cell renal cell carcinoma," Cancer, vol. 119, no. 2, pp. 313-324, 2013.

[18] H. Fujita, K. Ohuchida, K. Mizumoto et al., " $\alpha$-Smooth muscle actin expressing stroma promotes an aggressive tumor biology in pancreatic ductal adenocarcinoma," Pancreas, vol. 39, no. 8, pp. 1254-1262, 2010.

[19] E. Olaso, A. Santisteban, J. Bidaurrazaga, A. M. Gressner, J. Rosenbaum, and F. Vidal-Vanaclocha, "Tumor-dependent activation of rodent hepatic stellate cells during experimental melanoma metastasis," Hepatology, vol. 26, no. 3, pp. 634-642, 1997.

[20] Y. Zhang, H. Tang, J. Cai et al., "Ovarian cancer-associated fibroblasts contribute to epithelial ovarian carcinoma metastasis by promoting angiogenesis, lymphangiogenesis and tumor cell invasion," Cancer Letters, vol. 303, no. 1, pp. 47-55, 2011.

[21] X. Wu, X. Chen, Q. Zhou et al., "Hepatocyte growth factor activates tumor stromal fibroblasts to promote tumorigenesis in gastric cancer," Cancer Letters, vol. 335, no. 1, pp. 128-135, 2013.

[22] A. Popple, L. G. Durrant, I. Spendlove et al., "The chemokine, CXCL12, is an independent predictor of poor survival in ovarian cancer," British Journal of Cancer, vol. 106, no. 7, pp. 1306-1313, 2012.

[23] T. Shiozaki, T. Tabata, N. Ma et al., "Association of CXC chemokine receptor type 4 expression and clinicopathologic features in human vulvar cancer," International Journal of Gynecological Cancer, vol. 23, no. 6, pp. 1111-1117, 2013.

[24] B. C. Lee, T. H. Lee, S. Avraham, and H. K. Avraham, "Involvement of the chemokine receptor CXCR4 and its ligand stromal cell-derived factor $1 \alpha$ in breast cancer cell migration through human brain microvascular endothelial cells," Molecular Cancer Research, vol. 2, no. 6, pp. 327-338, 2004.

[25] J. Li, X. S. Zhang, D. Xie et al., "Expression of immune-related molecules in primary EBV positive Chinese nasopharyngeal carcinoma: associated with latent membrane protein 1 (LMP1) expression," Cancer Biology and Therapy, vol. 6, no. 12, pp. 19972004, 2007.

[26] N. Wang, Q. L. Wu, Y. Fang et al., "Expression of chemokine receptor CXCR4 in nasopharyngeal carcinoma: pattern of expression and correlation with clinical outcome," Journal of Translational Medicine, vol. 3, article 26, 2005.

[27] S. Saigusa, Y. Toiyama, K. Tanaka et al., "Cancer-associated fibroblasts correlate with poor prognosis in rectal cancer after chemoradiotherapy," International Journal of Oncology, vol. 38, no. 3, pp. 655-663, 2011.

[28] A. Orimo and R. A. Weinberg, "Stromal fibroblasts in cancer: a novel tumor-promoting cell type," Cell Cycle, vol. 5, no. 15, pp. 1597-1601, 2006

[29] L. F. Brown, A. J. Guidi, S. J. Schnitt et al., "Vascular stroma formation in carcinoma in situ, invasive carcinoma, and metastatic carcinoma of the breast," Clinical Cancer Research, vol. 5, no. 5, pp. 1041-1056, 1999. 
[30] D. Fukumura, R. Xavier, T. Sugiura et al., “Tumor induction of VEGF promoter activity in stromal cells," Cell, vol. 94, no. 6, pp. 715-725, 1998.

[31] R. E. Bachelder, A. Crago, J. Chung et al., "Vascular endothelial growth factor is an autocrine survival factor for neuropilinexpressing breast carcinoma cells," Cancer Research, vol. 61, no. 15, pp. 5736-5740, 2001.

[32] J. F. Liang, H. K. Wang, H. Xiao et al., "Relationship and prognostic significance of SPARC and VEGF protein expression in colon cancer," Journal of Experimental and Clinical Cancer Research, vol. 29, no. 1, article 71, 2010.

[33] P. O. van Trappen, D. Steele, D. G. Lowe et al., "Expression of vascular endothelial growth factor (VEGF)-C and VEGF-D, and their receptor VEGFR-3, during different stages of cervical carcinogenesis," Journal of Pathology, vol. 201, no. 4, pp. 544554, 2003.

[34] D. Lyden, K. Hattori, S. Dias et al., "Impaired recruitment of bone-marrow-derived endothelial and hematopoietic precursor cells blocks tumor angiogenesis and growth," Nature Medicine, vol. 7, no. 11, pp. 1194-1201, 2001.

[35] P. Carmeliet and R. K. Jain, "Angiogenesis in cancer and other diseases," Nature, vol. 407, no. 6801, pp. 249-257, 2000.

[36] B. Heissig, K. Hattori, S. Dias et al., "Recruitment of stem and progenitor cells from the bone marrow niche requires MMP-9 mediated release of Kit-ligand," Cell, vol. 109, no. 5, pp. 625-637, 2002.

[37] H. S. Hwang, Y. S. Maeng, Y. H. Kim, Y. G. Kwon, Y. W. Park, and I. K. Kim, "Nestin expression during differentiation of fetal endothelial progenitor cells and hypoxic culture of human umbilical vein endothelial cells," Acta Obstetricia et Gynecologica Scandinavica, vol. 87, no. 6, pp. 643-651, 2008.

[38] A. Patenaude, J. Parker, and A. Karsan, "Involvement of endothelial progenitor cells in tumor vascularization," Microvascular Research, vol. 79, no. 3, pp. 217-223, 2010.

[39] T. W. Chen, Z. G. Yang, Q. L. Wang, Y. Li, L. L. Qian, and H. J. Chen, "Whole tumour quantitative measurement of firstpass perfusion of oesophageal squamous cell carcinoma using 64-row multidetector computed tomography: correlation with microvessel density," European Journal of Radiology, vol. 79, no. 2, pp. 218-223, 2011.

[40] C. N. Qian, D. Huang, B. Wondergem, and B. T. Teh, "Complexity of tumor vasculature in clear cell renal cell carcinoma," Cancer, vol. 115, no. 10, pp. 2282-2289, 2009.

[41] G. Ö. Elpek, T. Gelen, N. H. Aksoy et al., "The prognostic relevance of angiogenesis and mast cells in squamous cell carcinoma of the oesophagus," Journal of Clinical Pathology, vol. 54, no. 12, pp. 940-944, 2001.

[42] A. Gadducci, P. Viacava, S. Cosio et al., "Intratumoral microvessel density, response to chemotherapy and clinical outcome of patients with advanced ovarian carcinoma," Anticancer Research $B$, vol. 23, no. 1, pp. 549-556, 2003.

[43] R. L. Foote, N. Weidner, J. Harris et al., "Evaluation of tumor angiogenesis measured with microvessel density (MVD) as a prognostic indicator in nasopharyngeal carcinoma: results of RTOG 9505," International Journal of Radiation Oncology Biology Physics, vol. 61, no. 3, pp. 745-753, 2005.

[44] S. E. Kountakis, Encyclopedia of Otolaryngology, Head and Neck Surgery, Springer, 2013.

[45] Y. Shaked and R. S. Kerbel, "Antiangiogenic strategies on defense: on the possibility of blocking rebounds by the tumor vasculature after chemotherapy," Cancer Research, vol. 67, no. 15, pp. 7055-7058, 2007. 


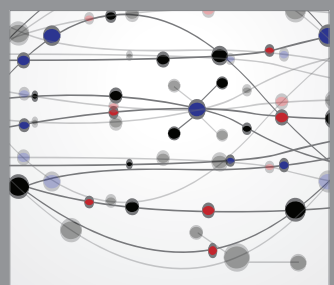

The Scientific World Journal
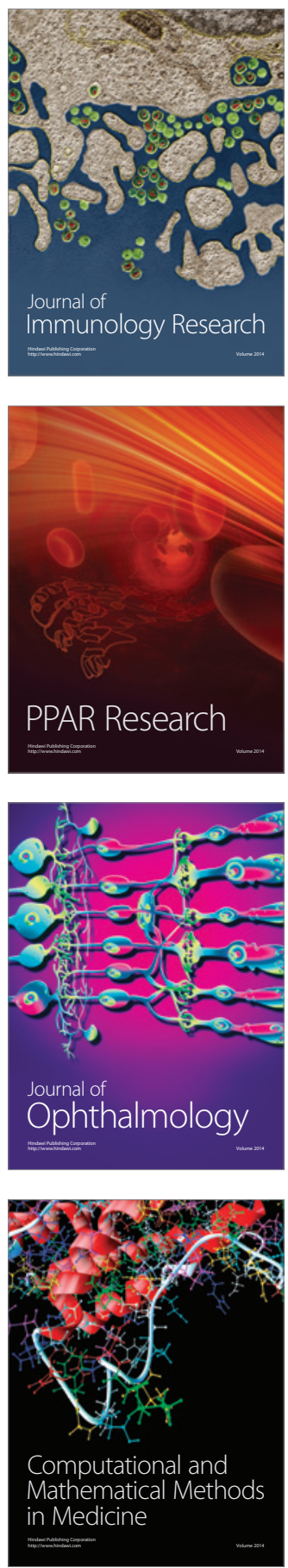

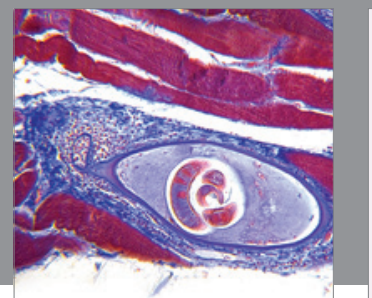

Gastroenterology

Research and Practice
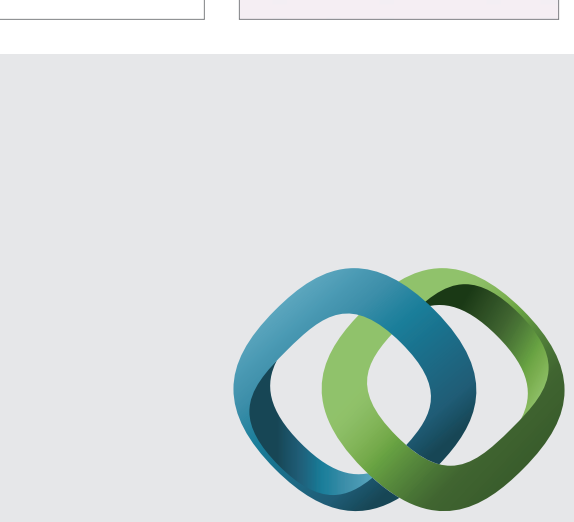

\section{Hindawi}

Submit your manuscripts at

http://www.hindawi.com
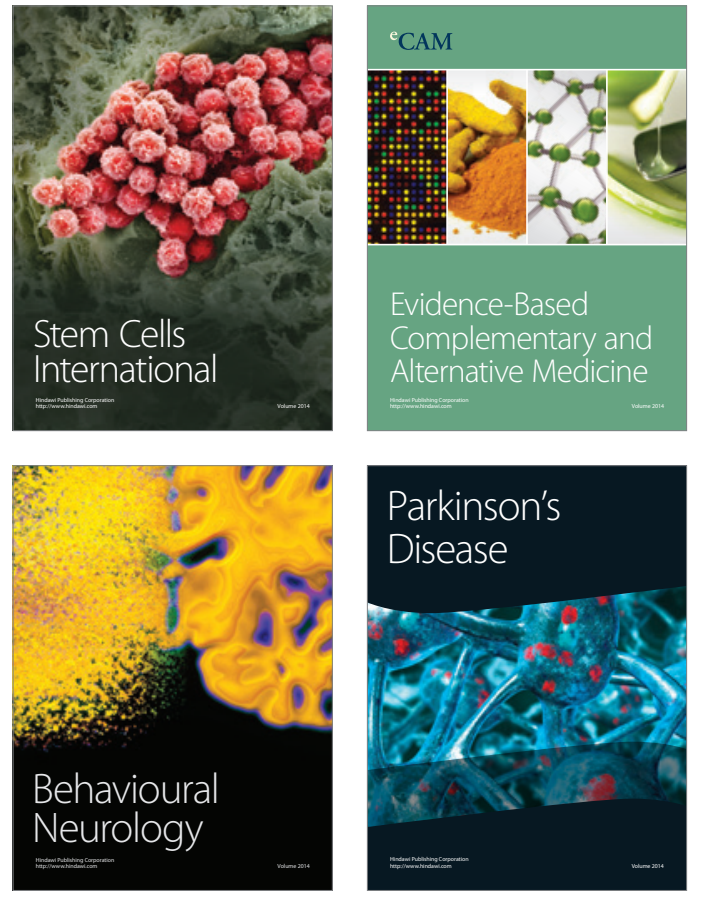
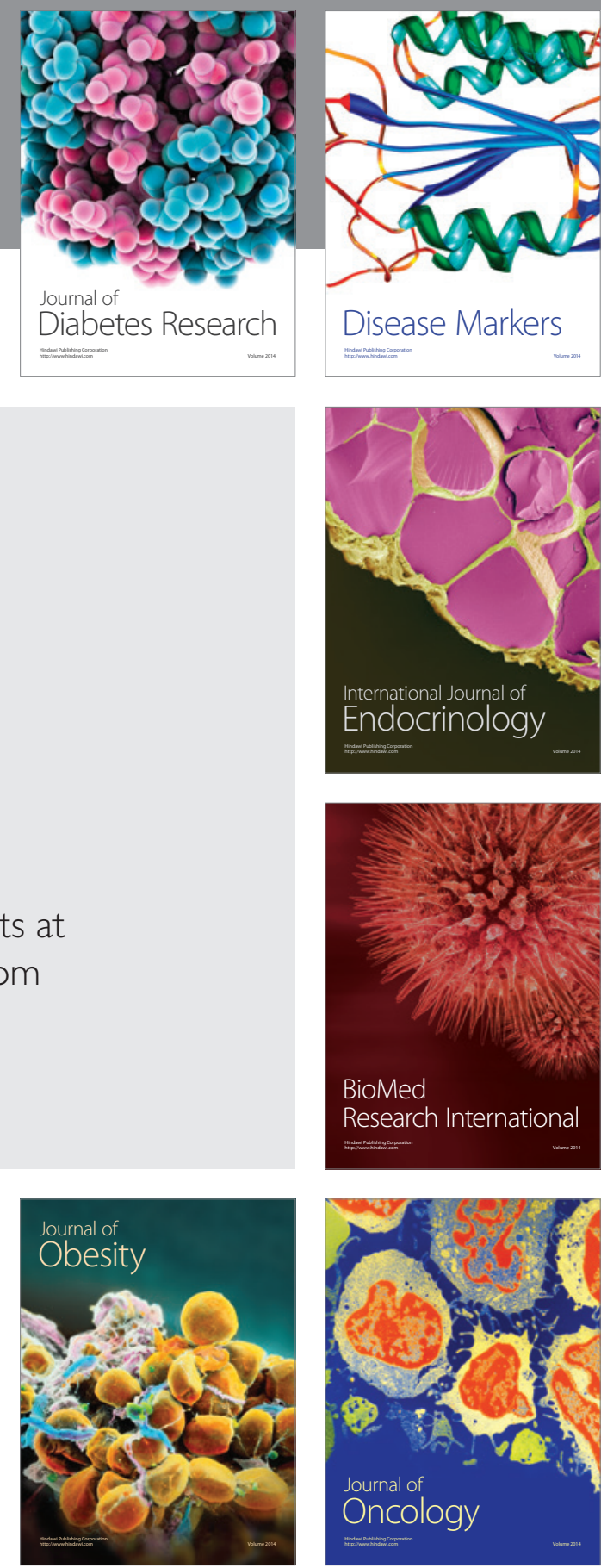

Disease Markers
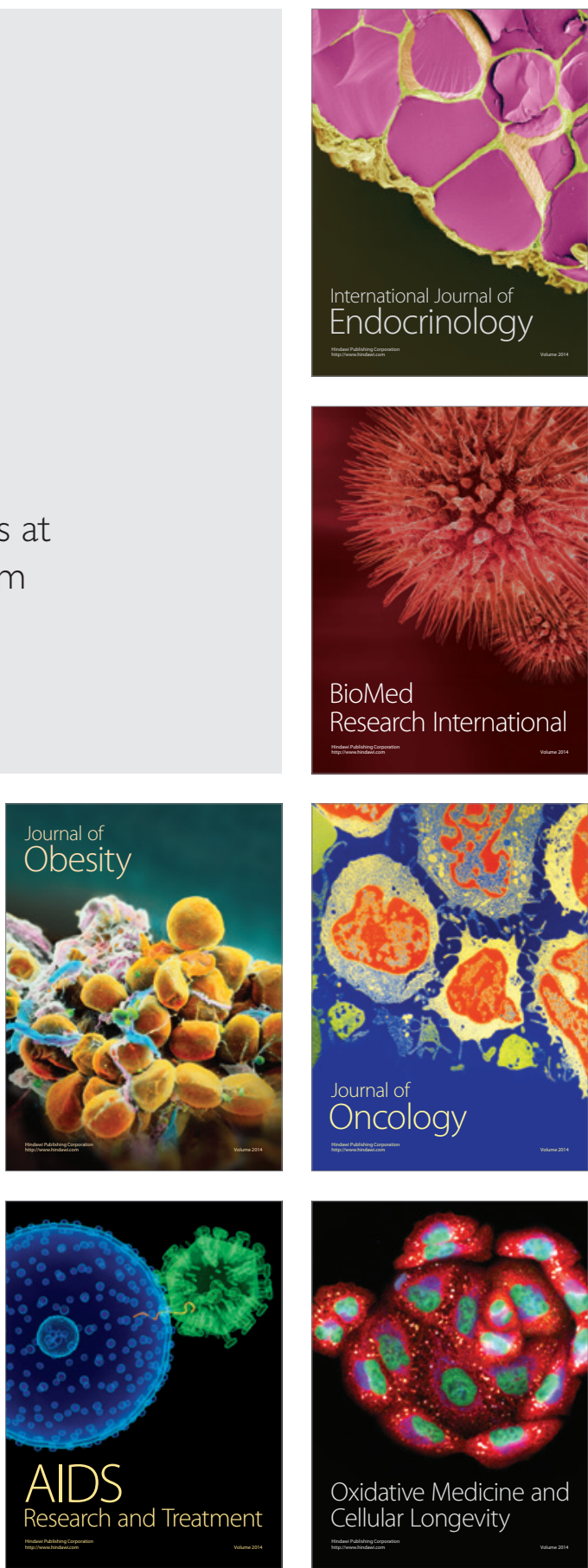